\title{
Necessidades fisioterapêuticas de idosos em atendimento ambulatorial
}

\author{
Mara Regina Knorst", Milena Abreu Tavares de Sousa Fischer", Ângelo Jose Gonçalves Bós"*
}

\section{Resumo}

O envelhecimento populacional tem aumentado a prevalência de doenças crônicas e incapacitantes, as quais levam à limitação funcional, diminuindo a autonomia e a qualidade de vida dos idosos. Limitações funcionais causam dependência para o desempenho de tarefas, ou seja, atividades de vida diária (AVD) e atividades instrumentais de vida diária (AIVD). A atenção ambulatorial tem aumentado para com os idosos, em vista da demanda por serviços ambulatoriais de reabilitação geriátrica, na qual o fisioterapeuta tem um papel relevante. Este estudo busca conhecer as principais demandas de atendimento fisioterapêutico em ambulatório geriátrico, propondo a estrutura ideal mínima desse serviço. Em um estudo transversal, descritivo e analítico, pacientes de um ambulatório de geriatria com sessenta anos ou mais, de ambos os sexos, foram convidados a participar da pesquisa. Os voluntários foram inquiridos sobre as AVD e AIVD e tiveram avaliadas as funções de equilíbrio, flexibilidade articular e função muscular. A presença de doenças, estado mental e depressão foi coletada por consulta ao prontuário médico. Os 55 idosos participantes tinham indicação de acompanhamento fisioterapêutico. Demência e gota foram significativas para perda de AVD e AIVD. Verificou-se também perda significativa para AIVD entre idosos com osteoartrose. Entre as técnicas fisioterapêuticas, a mais indicada foi a cinesioterapia motora, que inclui exercícios ativos passivos, resistidos e caminhada orientada. Conclui-se que as doenças crônicas estão associadas à dependência física e que o fisioterapeuta tem um papel importante na reabilitação funcional do idoso ambulatorial. Faz-se necessária a criação de espaço físico dentro de um serviço ambulatorial contendo equipamentos específicos da fisioterapia.

Palavras-chave: Fisioterapia. Reabilitação. Geriátrica. Autonomia. Qualidade de vida.

* Doutorado em Gerontologia Biomédica pela Pontifícia Universidade Católica do Rio Grande do Sul. Professor Titular da Pontifícia da Faculdade de Enfermagem Fisioterapia e Nutrição da Pontifícia Universidade Católica do Rio Grande do Sul.

* Graduação em Fisioterapia pela Universidade Estadual da Paraíba. Mestranda no Programa de PósGraduação em Gerontologia Biomédica da Pontifícia Universidade Católica do Rio Grande do Sul.

**** Doutorado em Medicina pela Tokai University, School of Medicine, Japão. Professor Adjunto da Pontifícia Universidade Católica do Rio Grande do Sul. Orientador no Programa de Pós-Graduação em Gerontologia Biomédica da Pontifícia Universidade Católica do Rio Grande do Sul. Endereço para correspondência: Av. Ipiranga, 6690, 3o andar, bairro Jardim Botânico, CEP: 90610-000, Porto Alegre - RS. E-mail: angelo.bos@ pucrs.br.

$\hookrightarrow$ Recebido em fevereiro de 2009 - Avaliado em agosto de 2009.

$\rightarrow$ doi:10.5335/rbceh.2010.002 


\section{Introdução}

O envelhecimento é um processo fisiológico benigno compatível com a independência para as atividades físicas. Entretanto, à medida que o indivíduo envelhece, o organismo se defronta com várias modificações morfológicas e funcionais, caracterizadas por atrofias e diminuição da eficiência funcional, tornando-o mais vulnerável a doenças. (NICOLA, 1986). Tanto é assim que a frequência de doenças crônicas aumenta com a idade. Oitenta por cento dos idosos apresenta, pelo menos, uma condição crônica. Um grande número de pessoas idosas apresenta algum tipo de limitação para a realização das atividades de vida diária e, com o aumento da idade, essas limitações tendem a aumentar (PASCHOAL, 1996), diminuindo a autonomia e, consequentemente, a qualidade de vida.

O aumento da população idosa deve despertar preocupação entre as autoridades, pois os idosos consomem mais recursos de saúde, utilizam mais os serviços e, consequentemente, custam mais para o Estado. Por conseguinte, as doenças crônicas obrigam seus portadores a procurar os serviços de saúde com grande frequência, havendo necessidade aumentada de recursos materiais e humanos, muitas vezes com tecnologia complexa. (PASCHOAL, 1996; WELLS et al., 2003). Investir em prevenção, não somente em nível primário, mas também no secundário e terciário, em centros qualificados aos idosos, aumentaria a autonomia e a qualidade de vida da pessoa idosa, diminuindo a relação do custo e desafogando o sistema prestador de serviço. (FORTENZA, 2000).

Nesse contexto, o fisioterapeuta é o profissional que promove a prevenção terciária, ou seja, a reabilitação funcional do idoso, buscando a recuperação das suas capacidades físicas dentro das suas limitações funcionais, permitindo sua integração com a sociedade e melhorando, dessa forma, a autonomia e a sua qualidade de vida.

As limitações funcionais levam o indivíduo à dependência para o desempenho de tarefas, bem como a défice no desempenho das atividades da vida diária (AVD) e das chamadas atividades instrumentais da vida diária (AIVD) (GUCCIONE, 2002). O termo AVD é utilizado na literatura científica como o conjunto de atividades do cotidiano relacionadas a banhar-se, alimentar-se, vestir-se, locomover-se, transferir-se e manter o controle esfincteriano, principalmente no contexto das avaliações e instrumentos de capacidade funcional. Já as AIVD se referem às atividades mais elaboradas, que requerem uma maior interação da pessoa com o meio em que vive, tais como preparar refeição, tomar seus remédios, fazer compras, controlar seu dinheiro, usar o telefone, arrumar sua casa, passar roupa, fazer pequenos trabalhos domésticos e sair de casa sozinho. (KATZ et al., 1983).

Com esse número crescente de pessoas idosas com doenças crônicas, a atenção ambulatorial tem aumentado nessa faixa etária, o que exige a participação maior de uma equipe multiprofissional. Há necessidade de se criarem serviços ambulatoriais de reabilitação geriátrica. 
Contudo, previamente à criação desse serviço ambulatorial, é importante terse definida a clientela a ser atendida, como também as necessidades dessa, definindo, assim, a estrutura básica desse serviço.

Dentro dessa realidade, o presente trabalho busca conhecer as demandas de atendimento fisioterapêutico num ambulatório geriátrico e propor a estrutura mínima deste serviço. Além disso, tem como objetivos observar as doenças mais prevalentes nessa população e a sua provável associação com o grau de dependência dos idosos; avaliar as necessidades da intervenção fisioterapêutica desses idosos; propor a estrutura mínima para o atendimento fisioterapêutico de uma unidade de atendimento ambulatorial geriátrico.

\section{Materiais e métodos}

O presente estudo foi um estudo transversal, descritivo e analítico, desenvolvido no período de junho de 2001 a fevereiro de 2002 no Ambulatório de Geriatria do Hospital São Lucas da PUCRS.

A população estudada constou de pacientes com sessenta anos ou mais, de ambos os sexos, que retornaram após a primeira consulta ao Ambulatório de Geriatria do Hospital São Lucas (HSL) e concordaram em participar da pesquisa após a explicação de seus objetivos e procedimentos. Feito isso, os participantes assinaram o termo de consentimento informado do projeto, que foi aprovado pelo Comitê de Ética em Pesquisa da PUCRS 24.04.2001, sob protocolo n. 01/910. Os instrumentos de coleta de dados foram desenvolvidos com base na ficha de avaliação utilizada no Serviço de Geriatria do HSL. A avaliação consistiu na aplicação dos questionários, pelo qual os voluntários foram inquiridos sobre as AVD e AIVD, hábitos de vida e motivos da consulta. Também foram avaliados quanto a funções de equilíbrio, flexibilidade articular e função muscular. Dados sobre a presença de doenças, estado mental, depressão, medicamentos foram coletados por meio da avaliação do prontuário e comunicação com o médico assistente.

Os questionários das AVD e AIVD estimaram as incapacidades que foram mais bem avaliadas pelos exames das funções de equilíbrio, flexibilidade articular e função muscular. A avaliação do equilíbrio consistiu de três testes, adaptados de Tineti e Jags (1986). No primeiro, foi solicitado ao paciente que se levantasse da cadeira, desse dez passos, fizesse a volta, retornasse e se sentasse novamente. Foi observado se o paciente apresentava dificuldade em executar a tarefa. No segundo, foi solicitado ao paciente que encostasse o ombro e braço direito estendido na parede e fosse inclinando o corpo para frente até que sentisse alguma perda de equilíbrio, observando-se a distância de deslocamento. Deslocamento inferior a $15 \mathrm{~cm}$ foi considerado alteração de equilíbrio. O terceiro teste consistiu no teste de Romberg, pelo qual o equilíbrio é testado com o paciente em pé e com os olhos fechados; no caso de alteração de equilíbrio considera-se a prova positiva. Por meio dos dois primeiros testes foram observadas alterações de marcha, perda 
de amplitude de movimento, coordenação motora e deformidades.

Dados como doenças atuais e doenças prévias foram coletados do prontuário do paciente e proporcionaram subsídios com relação a possíveis sequelas de doenças anteriores e doenças atuais que pudessem estar associadas. A avaliação cognitiva foi realizada por meio do teste do Mini-Estado Mental (MEM), e a depressão, pela escala de depressão geriátrica (Yesavage). Os resultados do Mini-Mental e da escala de depressão foram coletados do prontuário do paciente e auxiliaram numa melhor avaliação das condições físicas do idoso, como também na elaboração do tratamento fisioterapêutico.

Os testes da função dos braços distal e proximal foram utilizados para observar a amplitude de movimento e coordenação de membros superiores, bem como a preservação de força muscular. $\mathrm{O}$ primeiro teste consistiu em solicitar ao paciente que pegasse uma caneta com cada uma das mãos e a colocasse de volta na mesa, observando-se a presença de dificuldades. No segundo teste, orientavase o paciente a tocar a parte posterior da cabeça com ambas as mãos e, se ele apresentasse dificuldades, realizavamse testes específicos de função muscular e examinavam-se cuidadosamente as articulações envolvidas. A amplitude de movimento e a presença de deformidades foram avaliadas pela inspeção e com os testes de equilíbrio e função de braços distal e proximal. Os testes de força muscular foram realizados se o paciente apresentasse resultado positivo para alterações em algum dos outros testes realizados.
A observação da incontinência urinária foi importante, pois nos indicou o grau e o motivo da incontinência. $\mathrm{O}$ paciente foi inquirido se às vezes perdia urina ou ficava molhado. No caso de resposta negativa, o teste era considerado negativo e era interrompido. Caso a resposta fosse positiva, perguntava-se sobre a presença de dificuldades para segurar a urina, se perdia urina mesmo quando tentava evitar ou quando tossia, ria ou quando tinha urgência. Perguntava-se, ainda, a frequência em que esses episódios ocorriam, os horários, situação e quantidade.

Os dados coletados foram avaliados por meio de estatística descritiva. As AVD e AIVD foram utilizadas para medir o grau de dependência do grupo pesquisado. Pessoas com nível menor que seis são consideradas dependentes.

Analisados os dados, buscou-se associar as patologias mais prevalentes com as incapacidades mais frequentes na população estudada, pela criação de tabelas de distribuição. Com base na avaliação fisioterapêutica, traçou-se um programa de reabilitação fisioterapêutica adaptada às condições do grupo estudado.

\section{Resultados}

Durante o período da pesquisa, 212 idosos retornaram ao ambulatório após a primeira consulta. Conforme a disponibilidade da pesquisadora, uma vez por semana, em média, dois idosos foram entrevistados, ou seja, 72. Dados incompletos de prontuários ou a impossibilidade de uma avaliação completa 
por limite de tempo ou cansaço do idoso reduziram o tamanho amostral para 55 pacientes, totalizando $25,9 \%$. A média de idade foi de $73,0 \pm 13,07$ anos; $73 \%$, ou 40 participantes, eram mulheres, com idade média de $71,5 \pm 14,15$ quanto 15 participantes $(27 \%)$ eram homens com idade média de 77,2 \pm 8,66 anos.

A Tabela 1 mostra a distribuição em números absolutos e relativos das amostras quanto às doenças mais prevalentes entre idosos do ambulatório: hipertensão arterial sistêmica (HAS) $64 \%$, insuficiência cardíaca (IC) $22 \%$, osteoporose $20 \%$, osteoartrose de membros inferiores (OAMI) com 16\%, diabetes melitus (DM) $13 \%$, DPOC e obesidade com $9 \%$. Observaram-se ainda $5 \%$ com osteoartrose de membros superiores (OAMS), demência, depressão, fraturas de membros inferiores, gota, hiperplasia benigna de próstata, incontinência urinária e tendinite de membros superiores.

Tabela 1 - Frequência, média e desvio-padrão das doenças relacionadas com as AVD e AIVD.

\begin{tabular}{|c|c|c|c|c|c|c|c|c|c|c|}
\hline \multirow[t]{2}{*}{ Doenças } & \multicolumn{2}{|c|}{ Pessoas } & \multicolumn{2}{|c|}{$\begin{array}{c}\text { AVD } \\
\text { (com doença) }\end{array}$} & \multicolumn{2}{|c|}{$\begin{array}{c}\text { AIVD } \\
\text { (com doença) }\end{array}$} & \multicolumn{2}{|c|}{$\begin{array}{c}\text { AVD } \\
\text { (sem doença) }\end{array}$} & \multicolumn{2}{|c|}{$\begin{array}{c}\text { AIVD } \\
\text { (sem doença) }\end{array}$} \\
\hline & $n$ & $\%$ & Média & DP & Média & DP & Média & DP & Média & $\mathrm{DP}$ \\
\hline HAS & 35 & 64 & 5,2 & 1,76 & 6,1 & 2,5 & 5,6 & 1,46 & 6,4 & 2,45 \\
\hline IC & 12 & 22 & 5,7 & 0,86 & 6,6 & 2,6 & 5,2 & 1,8 & 6,1 & 2,43 \\
\hline Osteoporose & 11 & 20 & 6,0 & 0,77 & 6,3 & 2,3 & 5,2 & 1,78 & 6,2 & 2,52 \\
\hline OA MI & 9 & 16 & 5,8 & 0,33 & 7,2 & 1,6 & 5,3 & 1,79 & 6,1 & 2,57 \\
\hline DM & 7 & 13 & 5,1 & 2,26 & 5,8 & 1,9 & 5,4 & 1,58 & 6,3 & 2,53 \\
\hline DPOC & 5 & 9 & 5,8 & 0,44 & 5,6 & 2,2 & 5,3 & 1,73 & 6,3 & 2,49 \\
\hline Obesidade & 5 & 9 & 4,8 & 2,68 & 5,4 & 3,1 & 5,4 & 1,55 & 6,4 & 2,40 \\
\hline OAMS & 3 & 5 & $6,0^{\star}$ & 0,00 & 8,0 & 0,0 & 5,3 & 1,70 & 6,2 & 2,49 \\
\hline Demência & 3 & 5 & $3,6^{\star}$ & 3,21 & $2,6^{*}$ & 2,3 & 5,5 & 1,52 & 6,5 & 2,32 \\
\hline Depressão & 3 & 5 & 5,3 & 1,15 & 5,6 & 2,0 & 5,4 & 1,69 & 6,3 & 2,49 \\
\hline Fraturas MI & 3 & 5 & 5,5 & 0,57 & 6,7 & 2,5 & 5,3 & 1,71 & 6,2 & 2,47 \\
\hline Gota & 3 & 5 & $2,0^{\star}$ & 3,46 & $1,3^{*}$ & 2,3 & 5,5 & 1,31 & 6,5 & 2,16 \\
\hline HBP & 3 & 5 & 5,6 & 0,57 & 6,3 & 2,0 & 5,3 & 1,70 & 6,3 & 2,5 \\
\hline IU & 3 & 5 & 5,3 & 1,15 & 5,3 & 2,3 & 5,4 & 1,69 & 6,3 & 2,47 \\
\hline Tendinite MS & 3 & 5 & 5,2 & 1,76 & 5,3 & 4,6 & 5,4 & 1,52 & 6,3 & 2,34 \\
\hline
\end{tabular}

* $\mathrm{p}<0,05$ para teste $\mathrm{t}$ de Student comparando as médias das AVD e AIVD entre as pessoas com e sem a doença. OA = osteoartrose; $\mathrm{MI}=$ membros inferiores; $\mathrm{MS}$ membros superiores.

Na Tabela 1 as médias e desvio-padrão das AVD e AIVD foram calculados para cada patologia. Essas médias foram comparadas com as dos pacientes que não tinham as patologias. $\mathrm{O}$ teste $\mathrm{t}$ de Student, comparando ambas as médias, mostrou-se significativo na perda de AVD para artrose de membros superiores, demência, gota e na perda de AIVD para demência e gota. 
Tabela 2 - Procedimentos fisioterapêuticos mais indicados pela avaliação das 55 pessoas observadas no Ambulatório de Geriatria do HSL.

\begin{tabular}{lcccc}
\hline \multirow{2}{*}{ Técnicas utilizadas } & \multicolumn{2}{c}{ Observadas } & \multicolumn{2}{c}{ Estimadas } \\
\cline { 2 - 5 } & Indicações & Média de uso & Indicações & Média de uso \\
\hline Exercícios resistidos membros inferiores & 24 & 11,3 & 14,4 & 162,7 \\
Exercícios ativos membros inferiores & 22 & 9,9 & 13,2 & 130,7 \\
Exercícios ativos membros superiores & 20 & 7,9 & 12,07 & 95,3 \\
Treino de equilíbrio & 15 & 5,9 & 9,05 & 53,4 \\
Caminhada orientada & 12 & 10,4 & 7,24 & 75,3 \\
Treino de marcha & 11 & 6,8 & 6,64 & 45,1 \\
Cinesioterapia respiratória & 10 & 11,5 & 6,03 & 69,3 \\
Exercícios ativos assistidos membros superiores & 6 & 7,8 & 3,62 & 28,2 \\
Exercícios passivos membros inferiores & 6 & 7,3 & 3,62 & 26,5 \\
Exercícios ativos assistidos membros inferiores & 5 & 8,8 & 3,01 & 26,5 \\
Exercícios resistidos membros superiores & 5 & 9,4 & 3,01 & 28,3 \\
Exercícios passivos membros superiores & 5 & 7,2 & 3,01 & 21,7 \\
Massagem lombar & 5 & 9,0 & 3,01 & 27,1 \\
Massagem cervical & 5 & 6,0 & 3,01 & 18,0 \\
\hline
\end{tabular}

Observam-se na Tabela 2 os procedimentos a serem realizados pelo profissional fisioterapeuta mais indicados para as 55 pessoas avaliadas no Ambulatório de Geriatria. Pode-se observar também a média de indicações quando estimados 32 atendimentos diários. Entre os procedimentos mais indicados para os
32 atendimentos no Ambulatório de Geriatria com o tempo diário estimado têm-se exercícios resistidos de membros inferiores, exercícios ativos de membros inferiores, exercícios ativos de membros superiores, caminhada orientada, cinesioterapia respiratória, treino de equilíbrio e treino de marcha.

Tabela 3 - Fisioterapêuticos mais frequentemente indicados no Ambulatório de Geriatria do HSL.

\begin{tabular}{lcccc}
\hline \multirow{2}{*}{ Técnicas utilizadas } & \multicolumn{2}{c}{ Observadas } & \multicolumn{2}{c}{ Estimadas } \\
\cline { 2 - 5 } & Pessoas & Média de uso & Indicações & Média de uso \\
\hline Tatame & 28 & 18,3 & 16,90 & 309,3 \\
Maca & 25 & 33,4 & 15,09 & 504,0 \\
Faixa elástica vermelha & 25 & 5,4 & 15,09 & 81,5 \\
Escada p/ maca & 22 & 37,0 & 13,28 & 491,4 \\
Bola & 21 & 5,3 & 12,67 & 67,1 \\
Cadeira & 20 & 25,6 & 12,07 & 309,0 \\
TENS / Cabo & 15 & 21,3 & 9,05 & 192,7 \\
Bastão & 13 & 5,8 & 7,84 & 45,5 \\
Infravermelho de pedestal & 12 & 18,8 & 7,24 & 136,1 \\
Bicicleta estacionária & 11 & 14,3 & 6,64 & 94,9 \\
Caneleiras 1/2 Kg & 9 & 6,1 & 5,43 & 33,1 \\
Cunha & 6 & 9,8 & 3,62 & 35,5 \\
Halteres 1/2 Kg & 6 & 4,8 & 3,62 & 17,4 \\
Nebulizador & 6 & 15,0 & 3,62 & 54,3 \\
Bolsa gel frio & 4 & 16,3 & 2,41 & 39,3 \\
Jogo de encaixe & 4 & 4,8 & 2,41 & 11,6 \\
Laser AsGa & 4 & 4,0 & 2,41 & 9,6 \\
Ondas curtas atérmico & 4 & 20,0 & 2,41 & 48,2 \\
Esteira computadorizada & 3 & 20,0 & 1,81 & 36,2 \\
\hline
\end{tabular}


Os equipamentos mais indicados para as 55 pessoas observadas no Ambulatório de Geriatria do HSL podem ser vistos na Tabela 3. Entre os mais indicados estão: maca, escada, tatame, cadeira, TENS, infravermelho de pedestal, bicicleta estacionária, faixa elástica, bola, nebulizador, bastão, bolsa gel fria, esteira computadorizada, cunha, caneleiras $1 / 2 \mathrm{~kg}$.

\section{Discussão}

Com o crescente aumento do envelhecimento populacional, as doenças crônicas, deficiências e incapacidades mais presentes em idosos passam a ocupar papel importante para os profissionais da saúde. (ELROD; DEJONG, 2008; PAULA, 2001).

Estudos em nível nacional pelo Sistema Único de Saúde concluíram que o idoso consome mais recursos que as pessoas mais jovens, não podendo ser traduzido como maior benefício para os idosos. Entre os problemas mais importantes para a terceira idade aparecem as dificuldades nas atividades de vida diária e o défice nutricional. (SILVESTRE, 1996).

Hoje a reabilitação na terceira idade oferece muitos recursos em nível da prevenção e tratamento. (PAULA, 2001). A reabilitação tem em vista a preservação da função para o adiamento da instalação de incapacidades, diminuir o comprometimento imposto por incapacidade e promover melhor adaptação do indivíduo, melhorando a sua qualidade de vida. (YUASO et al., 1996).

Entre as 55 pessoas avaliadas nesta pesquisa, observou-se a necessidade de acompanhamento fisioterapêutico para todos os idosos. Isso se deve ao fato de que a prevenção é importante, principalmente em idosos com alguma patologia que, somada com as alterações do envelhecimento, pode levar a uma diminuição significativa de suas possibilidades de mobilização e independência.

Entre os participantes do estudo realizado constatou-se maior incidência das doenças crônico-degenerativas, não restritas a um único órgão ou sistema. Em análise realizada por Lampert em 2002, com dados do Conselho Estadual do Idoso de 1997, observou-se na cidade de Santa Maria uma grande prevalência de doenças crônico-degenerativas em pessoas pesquisadas em seus domicílios. (LAMPERT, 2002). Pesquisas mostram as doenças crônico-degenerativas, entre as quais doenças cardiovasculares, diabetes e hipertensão arterial sistêmica, como causas importantes de incapacidade no idoso. (PINSKY et al., 1990). Entre os 408 idosos estudados em Santa Maria foi observado que pouco mais de um terço da amostra necessita de auxílio para realizar as AVD e um número menor necessita de auxílio para realizar AIVD. (LAMPERT, 2002).

No presente estudo observou-se que demências, entre as quais a doença de Alzheimer, foram significativas para perda de AVD e AIVD, tanto para internação quanto para o ambulatório. Demências são responsáveis por 50\% das admissões em asilos por causarem incapacidade. (IZZO et al., 2000). É importante levar em consideração o estágio da doença, como alterações na memória, na orientação, na percepção e nos conceitos, pois 
essas alterações são responsáveis pelas dificuldades motoras dos pacientes. Pacientes demenciados precisam de estímulo; caso contrário, permanecerão horas sentados. Esses pacientes não conseguem elaborar o ato de levantar, tampouco conseguem saber a finalidade deste ato. A inatividade leva à perda da força muscular, acarretando muitas vezes com limitações articulares e, se os pacientes dementados ficarem restritos ao leito, apresentarão deformidades.

Verificou-se perda significativa para AIVD entre os idosos portadores de osteoartrose, doença caracterizada por dor à movimentação, rigidez matinal, instabilidade articular, dor no repouso e limitação à movimentação, podendo levar à incapacidade. (YUASO et al., 1996).

Idosos geralmente apresentam gota na forma crônica, mais frequente no sexo masculino. Acomete mais as articulações dos joelhos, tornozelos e ombros, podendo levar à inflamação da membrana sinovial (sinovite), causando dor, diminuição de movimento e muitas vezes limitações (MEIRELLES, 2000), dados também comprovados em nosso estudo, uma vez que se encontrou perda significativa para AVD e AIVD entre os participantes da pesquisa.

Estudos mostram que $60 \%$ da população adulta portadora de limitação funcional, consequente a processos crônicos, têm mais de 65 anos. (CHIOVATTO, 1996). DPOC e pneumonia são as doenças respiratórias mais frequentes entre os idosos, levando à dispneia e à intolerância ao exercício, o que deixa os pacientes restritos, debilitados e muitas vezes incapacitados. Pneumonia é a causa de morte mais comum nos pacientes idosos. (YUASO et al., 1996). No estudo realizado observou-se perda significativa para AIVD entre os participantes portadores de pneumonia da internação geriátrica.

Entre as técnicas fisioterapêuticas mais indicadas aos pacientes observados tem-se a cinesioterapia motora, na qual se incluem exercícios ativos passivos, resistidos e caminhada orientada. Esta última vislumbra pacientes sem incapacidades, como os portadores de HAS e osteoporose, nos quais o principal problema é o sedentarismo, pois com o envelhecimento ocorrem atrofias, advindo, assim, a necessidade da realização de exercícios de forma regular e progressiva, que objetivam o retardo das atrofias e degenerações. (YUASO et al., 1996). Entre as técnicas mais indicadas há ainda a cinesioterapia respiratória, responsável tanto pela prevenção de doenças como pelo seu tratamento. Com indicações em menor número vislumbrase a massagem, objetivando relaxamento muscular, aumento de retorno venoso, mobilização de edemas e melhora em processos fibróticos. (PEREIRA et al., 2001).

Entre os idosos avaliados no estudo, constatou-se maior indicação para os equipamentos utilizados na cinesioterapia motora, como bola, faixas elásticas, bastão; na eletroterapia, o uso do TENS e, na termoterapia, o uso do infravermelho. $\mathrm{O}$ uso do infravermelho está particularmente indicado no idoso por ser calor superficial, que apresenta menores contraindicações. O TENS e o 
infravermelho são recursos utilizados para proporcionar analgesia, precedendo, em muitos casos, a cinesioterapia motora. Pesquisa realizada sobre sensibilidade dolorosa com uso de calor e gelo em mulheres jovens e idosas constatou alteração de sensibilidade entre estas duas faixas etárias. Mostrou-se que as idosas referiam apenas desconforto mínimo, ao passo que o desconforto referido pelas mulheres mais jovens era significativamente mais intenso. Diferenças foram observadas também em relação ao desconforto, quando comparados grupos de diferentes faixas etárias com relação ao uso da estimulação elétrica. (HAKANSSON et al., 1991).

Algumas diferenças foram observadas no ambulatório quanto à necessidade de equipamentos permanentes, visto que se necessita de tatame, macas, escadas para as mesmas e cadeiras para a viabilização dos tratamentos, recursos estes desnecessários na internação, em que o atendimento dar-se-á no leito do paciente. Ainda se observou, para o ambulatório, a necessidade de equipamentos como bicicleta estacionária, esteira computadorizada, laser, ondas curtas e ultrassom por se tratar de pacientes estáveis e com doenças crônicas.

\section{Conclusão}

A reabilitação tem papel importante na preservação da função, tendo em vista prevenir ou adiar a instalação de incapacidades, diminuindo, assim, o comprometimento imposto por enfermidades incapacitantes e promovendo uma melhor adaptação do indivíduo.
A prevenção deve ter destaque importante, sobretudo em idosos com alguma patologia que, somada às alterações do envelhecimento, pode levar a uma diminuição significativa de suas possibilidades de mobilização e independência. Quase sempre os pacientes idosos precisam de estímulo e orientação para a manutenção de sua independência. Caso contrário, permanecerão horas sentados, perdendo força muscular, ficando muitas vezes com limitações articulares, ou, se ficarem restritos ao leito, apresentarão deformidades.

A situação atual do envelhecimento brasileiro exige um melhor aprendizado das peculiaridades anatômicas e funcionais do envelhecimento. Devem-se formar profissionais que saibam discernir os efeitos naturais das alterações produzidas pelas inúmeras doenças que acometem o idoso e proporcionar treinamento específico na forma de abordagem e atendimento desta população.

Conclui-se que as doenças crônicas estão associadas à dependência física $\mathrm{e}$ que o fisioterapeuta tem papel importante na reabilitação funcional do idoso no atendimento ambulatorial. Faz-se, assim, necessária a criação de espaço físico dentro de um serviço ambulatorial contendo equipamentos específicos da fisioterapia. A eficácia do serviço proposto comprovaria a necessidade da criação desse espaço. 


\section{Physiotherapeutic needs of elderly people in ambulatory care}

\section{Abstract}

Aging population increases both chronic and disabling diseases. Chronic and disabling diseases lead to functional loss, decreased autonomy and quality of life. Functional loss increases the dependency for accomplishing tasks such as activity of daily living (ADL) and instrumental activities of daily living (IADL). Ambulatory care has increased for the elderly pressuring for more rehabilitation care in outpatient settings, demanding for the participation of the physiotherapist. This study aims to understand the main needs for physiotherapy in the elderly outpatient proposing the minimum conditions for this service. In a cross-sectional, observational and analytical approach, patients for an outpatient geriatric service, 60 years and older, both genders, participated in this study. The study group answered about the ADL and IADL, tested for balance, flexibility and muscle function. Prevalence of diseases, cognitive status, and depression were obtained from the medical charts. All 55 participants could benefit from a physiotherapic intervention. Dementia and gout associated significantly with both ADL and IADL. Osteoarthritis was significantly associated with IALD. Among the physiotherapic techniques, cinesioterapia was the most indicated. Cinesioterapia accounts for passive and active exercises, exercises against resistance and oriented walking. We concluded that chronic diseases are related with physical dependence and the physiotherapist was an important role in the functional rehabilitation in the elderly patient in an outpatient service. It is important to create a fully equipped physiotherapic yard in a geriatric outpatient service.

Key words: Physical. Therapy. Rehabilitation. Geriatric. Autonomy. Quality of life.

\section{Referências}

CHIOVATTO, J. Reabilitação em geriatria. In: PAPALÉO NETTO, M. Gerontologia: a velhice e o envelhecimento em visão globalizada. São Paulo: Atheneu, 1996. p. 324-330.

ELROD, C. S.; DEJONG, G. Determinants of utilization of physical rehabilitation services for persons with chronic and disabling conditions: an exploratory study. Arch. Phys. Med. Rehabil., v. 89, n. 1, p. 114-120, 2008.

FORTENZA, V. O. C. Neuropsiquiatria geriátrica. São Paulo: Atheneu, 2000.

GUCCIONE, A. O Estado de Saúde: estrutura conceitual e terminologia para exame, avaliação e diagnóstico. In: GUCCIONE, A. Fisioterapia geriátrica. Rio de Janeiro: Guanabara Koogan, 2002. p. 105-113.

HAKANSSON, D. et al. Effect of cold therapy on nerve conduction in young and elderly women. Physioterapy, v. 43, n. 2, 1991.

IZZO, H. et al. Fisioterapia. In: PAPALÉO NETTO, M. Geriatria: fundamentos, clínica e terapêutica. São Paulo: Atheneu, 2000. p. 393-397.

KATZ, S. Assessing self-maintenance: activities of daily living, mobility and instrumental activities of daily living. Journal of the American Geriatrics Society, v. 31, n. 12, p. 721-727, 1983.

LAMPERT, M. A. Avaliação multidimensional de idosos como preditora de risco de resultados adversos de saúde. 2002. 267 f. Tese (Doutorado em Clínica Médica e Ciências da Saúde) - Faculdade de Medicina da Pontifícia Universidade Católica do Rio Grande do Sul, Porto Alegre, 2002.

MEIRELLES, E. Principais artropatias e conectivopatias. In: CARVALHO FILHO, M. Geriatria: fundamentos, clínica e terapêutica. São Paulo: Atheneu, 2000, p. 309-317.

NICOLA, P. Manual de geriatria. Porto Alegre: L Editores, 1986. 
PASCHOAL, S. Epidemiologia do envelhecimento. In: PAPALÉO NETTO, M. Gerontologia: a velhice e o envelhecimento em visão globalizada. São Paulo: Atheneu, 1996. p. 26-43.

PAULA, J. A Reabilitação do idoso. In: LIANZA, S. Medicina de reabilitação. Rio de Janeiro: Guanabara Koogan, 2001. p. 410-419.

PEREIRA, C. et al. Reabilitação da mão. In: LIANZA, S. Medicina de reabilitação. Rio de Janeiro: Guanabara Koogan, 2001. p. 224-240.

PINSKY, J. et al. The framingham disability study: relationship of various coronary heart disease manifestations to disability in older persons, living in the community. Am. J. Public. Health., v. 80, n. 11, p. 1363-1367, Nov. 1990.

SILVESTRE, J. O Envelhecimento populacional brasileiro e o setor saúde. Arquivos de Geriatria e Gerontologia, v. 0, n. 1, p. 81-89, 1996.

WELLS, J. L. et al. State of the art in geriatric rehabilitation. Part I: review of frailty and comprehensive geriatric assessment. Arch. Phys. Med. Rehabil., v. 84, n. 6, p. 890-897, 2003.

YUASO, D. R. S. et al. Fisioterapia em pacientes idosos. In: PAPALÉO NETTO, M. Gerontologia: a velheice e o envelhecimento em visão globalizada. São Paulo: Atheneu, 1996. p. 331-347. 